\title{
A Representation for the Modules of a Graph and Applications
}

\author{
Sulamita Klein \\ Instituto de Matemática and COPPE/Sistemas, UFRJ \\ CP 68511, 21945-970 - Rio de Janeiro, RJ, Brazil \\ e-mail:sula@cos.ufrj.br
}

\author{
Jaime L. Szwarcfiter \\ Instituto de Matemática NCE and COPPE/Sistemas, UFRJ \\ CP 2324, 20001-970 - Rio de Janeiro, RJ, Brazil \\ e-mail: jayme@nce.ufrj.br
}

\begin{abstract}
We describe a simple representation for the modules of a graph $G$. We show that the modules of $G$ are in one-to-one correspondence with the ideals of certain posets. These posets are characterized and shown to be layered posets, that is, transitive closures of bipartite tournaments. Additionaly, we describe applications of the representation. Employing the above correspondence, we present methods for solving the following problems: (i) generate all modules of $G$, (ii) count the number of modules of $G$, (iii) find a maximal module satisfying some hereditary property of $G$ and (iv) find a connected non-trivial module of $G$.
\end{abstract}

Keywords: graphs, ideals, modules, posets, bipartite tournaments, algorithms

\section{Introduction}

There exists a vast literature on the study of modules of a graph, since this concept was first introduced by Gallay [5]. See [2, 3, 6, 7, 8, 12, 13]. In special there are several relevant algorithmic applications of the modular decomposition of a graph $[1,9,10,11,14]$. This paper describes a simple representation for the modules of a graph $G$. We show that the modules of $G$ are in one-to-one correspondence with the ideals of certain posets. These posets are shown to be transitive closures of bipartite tournaments.

$G$ denotes an undirected graph, $V(G)$ and $E(G)$ the vertex and edge sets of $G$, respectively, with $|V(G)|=$ $n$ and $|E(G)|=m$. For $v \in V(G), N_{G}(v)$ denotes the set of neighbours of $v$ in $G$, and $N_{G}[v]=N_{G}(v) \cup\{v\}$.
Let $\bar{N}_{G}(v)=V(G) \backslash N_{G}[v]$. A module of $G$ is a nonempty subset $M \subseteq V(G)$, such that every vertex $v \in$ $V(G) \backslash M$ is either a neighbour of all the vertices of $M$ or of none of them. Clearly, $V(G)$ and all one vertex subsets of $G$ are modules of $G$, called trivial modules. Finally, define a bipartite tournament as an orientation of a complete bipartite graph.

$D$ denotes a directed graph, or digraph, $V(D)$ and $E(D)$ are its sets of vertices and directed edges, respectively. For $v \in V(G)$, let $N_{D}^{+}(v)=\{w \in$ $V(D) \mid(v, w) \in E(D)\}, N_{D}^{-}(v)=\{w \in V(D) \mid(w, v) \in$ $E(D)\}, N_{D}^{+}[v]=N_{D}^{+}(v) \cup\{v\}$ and $N_{D}^{-}[v]=N_{D}^{-}(v) \cup$ $\{v\}$. Additionally, define $N_{D}(v)=N_{D}^{+}(v) \cup N_{D}^{-}(v)$ and $N_{D}[v]=N_{D}(v) \cup\{v\}$. When convenient we may drop the symbol of the graph or digraph, in the indices of these notations. If $N_{D}^{-}(v)=\emptyset$ then $v$ is the source of $D$. For $v, w \in V(D)$, if $D$ contains a $v-w$ path, then $v$ is an ancestor of $w$ and $w$ is a descendant of $v$. Say that $D$ is strongly connected when it contains both $v-w$ and $w-v$ paths, for every pair $v, w \in V(D)$. The strongly connected components of $D$ are the maximal strongly connected subdigraphs of $D$. The condensation $C$ of $D$ is the digraph whose vertices correspond to the strongly connected components of $D$, while $w \in N_{C}^{+}(v)$ when there is an edge in $D$ from a vertex lying in the strongly connected component corresponding to $v$, to a vertex in the component corresponding to $w$.

A digraph is transitive when $(v, w),(w, z) \in E(D)$ implies $(v, z) \in E(D)$, for all $v, w, z \in V(D)$. The transitive closure of a digraph $D$ is the transitive spanning superdigraph of $D$, preserving its reachability. A partially ordered set (poset) is an acyclic transitive digraph. An ideal of a poset $P$ is a subset $I \subseteq V(P)$, such that $x \in I$ implies $N_{D}^{-}(x) \subseteq I$, for all $x \in \bar{\epsilon} V(P)$.

Section 2 presents the correspondence between 


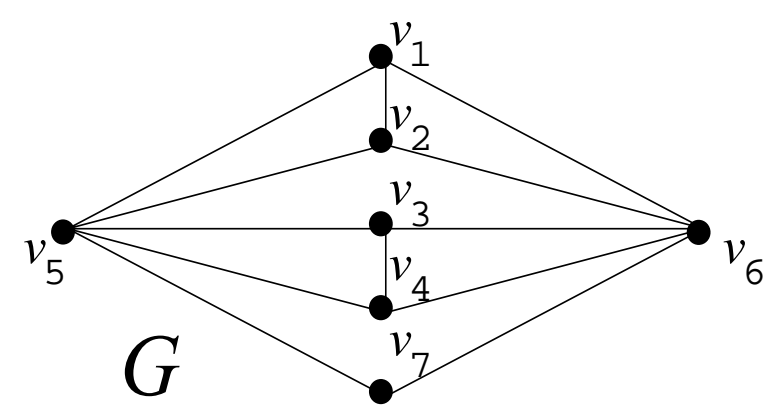

Figure 1: Graph G.

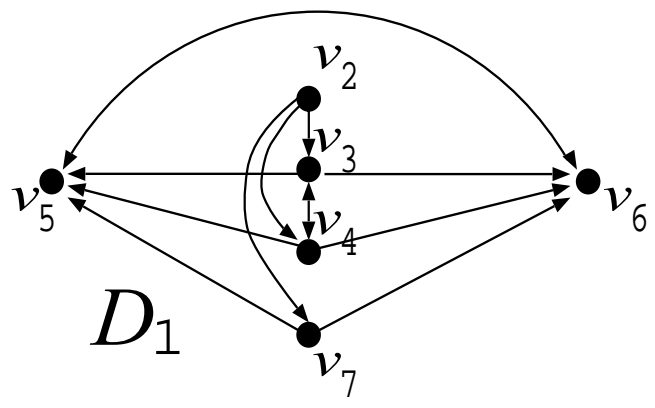

Figure 2: The modular digraph $D_{1}$ of $G$ relative to $v_{1}$.

modules of a graph $G$ and ideals of certain posets, as well as a characterization of these posets.

\section{The Representation}

Let $G$ be a graph and $v_{i} \in V(G)$. The modular digraph $D_{i}$ of $G$ relative to $v_{i}$, is one with vertex set $V(G) \backslash\left\{v_{i}\right\}$ and edge set defined as follows. For each pair of distinct vertices $v_{j}, v_{k} \in V(G), i \neq j, k$,

$$
\begin{aligned}
& \left(v_{j}, v_{k}\right) \in E(G) \Rightarrow \\
& \left\{\begin{array}{l}
\text { if } v_{j}, v_{k} \notin N_{G}\left(v_{i}\right) \\
\text { then }\left(v_{j}, v_{k}\right),\left(v_{k}, v_{j}\right) \in E\left(D_{i}\right) . \\
\text { if } v_{j} \notin N_{G}\left(v_{i}\right) \text { and } v_{k} \in N_{G}\left(v_{i}\right) \\
\text { then }\left(v_{j}, v_{k}\right) \in E\left(D_{i}\right) .
\end{array}\right. \\
& \left(v_{j}, v_{k}\right) \notin E(G) \Rightarrow \\
& \left\{\begin{array}{l}
\text { if } v_{j}, v_{k} \in N_{G}\left(v_{i}\right) \\
\text { then }\left(v_{j}, v_{k}\right),\left(v_{k}, v_{j}\right) \in E\left(D_{i}\right) . \\
\text { if } v_{j} \notin N_{G}\left(v_{i}\right) \text { and } v_{k} \in N_{G}\left(v_{i}\right) \\
\text { then }\left(v_{k}, v_{j}\right) \in E\left(D_{i}\right) .
\end{array}\right.
\end{aligned}
$$

$D_{i}$ contains no other edges, besides those as above.

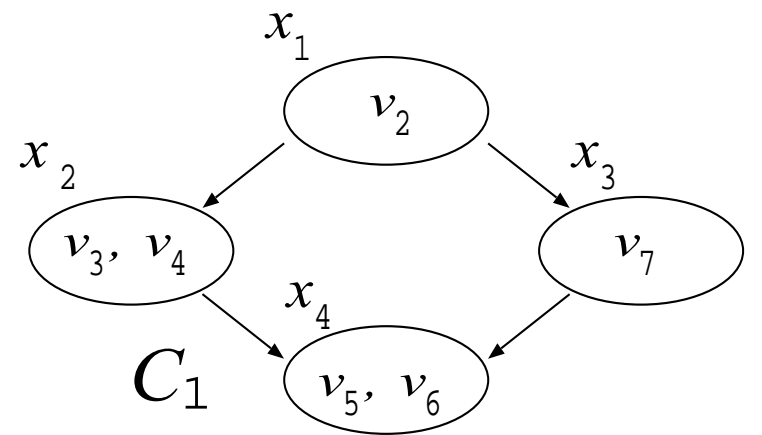

Figure 3: The condensation graph $C_{1}$ of $D_{1}$.

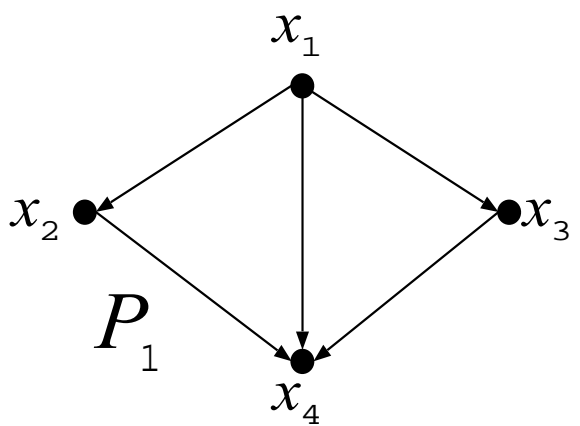

Figure 4: The modular poset $P_{1}$ of $G$ relative to $v_{1}$.

Further, define the modular poset $P_{i}$ of $G$, relative to $v_{i} \in V(G)$, as the transitive closure of the condensation $C_{i}$ of $D_{i}$. See Figures $1-4$ for examples.

Let $v \in V\left(D_{i}\right)$. Denote by $S(v) \subseteq V\left(D_{i}\right)$ the subset of vertices belonging to the same strongly connected component of $D_{i}$, as $v$ does. Similarly, for $x \in V\left(P_{i}\right)$, $S(x) \subseteq V\left(D_{i}\right)$ is the subset of vertices which forms the strongly connected component of $D_{i}$, corresponding to $x$ in the condensation. Call $S(v)$ the expansion of $v$, while $v$ is the reduction of $S(v)$ in $D_{i}$. On the other hand, $S(x)$ is the expansion of $x \in V\left(P_{i}\right)$ and $x$ is the reduction of $S(x)$ in $P_{i}$.

Ehrenfeucht et al. [4] have previously employed the condensation of a digraph (different from the above) in the process of computing the modular decomposition tree of a symmetric two-structure.

The following theorem characterizes the modules of $G$, in terms of the ideals of modular posets.

Theorem 1 Let $G$ be a graph, $v_{i} \in V(G)$ and $P_{i}$ the modular poset of $G$, relative to $v_{i}$. Then there exists a one-to-one correspondence between the ideals of $P_{i}$ and the modules of $G$ containing $v_{i}$. 
Proof: : Let $M$ be a module of $G$ containing $v_{i} \in$ $V(G)$. We show that we can always choose a convenient ideal $I$ of the modular poset $P_{i}$ of $G$, to correspond to $M$. Let $D_{i}$ be the modular digraph of $G$. We know that the vertices of $P_{i}$ are in one-to-one correspondence with the strongly connected components of $D_{i}$. Let $x$ be a vertex of $P_{i}$ and $S(x)$ the expansion of $x$.

Proposition 2 Either all vertices of $S(x)$ belong to $M$ or none of them does.

Proof: To start, note that the proposition is trivially true when $|S(x)|=1$. When $|S(x)|>1$, assume that it is false. In this case, $S(x)$ contains at least one vertex that belongs to $M$, and at least one which does not. Because the vertices of $S(x)$ form a strongly connected component of $D_{i}$, it follows that there must be an edge $\left(v_{j}, v_{k}\right) \in E\left(D_{i}\right)$, such that $v_{j}, v_{k} \in S(x)$, $v_{j} \notin M$ and $v_{k} \in M$. Examine the following alternatives for the pair of vertices $v_{j}, v_{k}$. First, assume that $\left(v_{j}, v_{k}\right) \in E(G)$. Then $v_{k} \in M$ and $v_{j} \notin M$ implies that $v_{j} \in N_{G}\left(v_{i}\right)$. In this case, (1.1) and (1.2) assure that $\left(v_{j}, v_{k}\right) \notin E\left(D_{i}\right)$. The other possibility is $\left(v_{j}, v_{k}\right) \notin E(G)$. In such situation, the implication is that $v_{j} \notin N_{G}\left(v_{i}\right)$. By applying this time, (2.1) and (2.2) we again conclude that $\left(v_{j}, v_{k}\right) \notin E\left(D_{i}\right)$, contradicting the fact that $\left(v_{j}, v_{k}\right)$ must be an edge of $D_{i}$. Hence Proposition 2 is true.

The proof of the theorem proceeds by assigning a label 0 or 1 to each vertex $x$ of $P_{i}$, as follows. Examine the set $S(x)$. By Proposition 2, either all vertices of $S(x)$ belong to $M$, or none of them does. In the former alternative, assign the label 0 to $x$. In the latter, assign 1 . Let I be the subset of vertices of $P_{i}$ having label 0 . In other words, $M=\left\{v_{i}\right\} \bigcup_{x \in I} S(x)$. We show that the subset $I$ is an ideal of $P_{i}$. If possible choose two vertices $x, y$ of $P_{i}$, such that $x$ is labelled $1, y$ has label 0 and $(x, y) \in E\left(P_{i}\right)$. Since $P_{i}$ is the transitive closure of the condensation of $D_{i}$, the latter digraph must contain an edge $\left(v_{j}, v_{k}\right) \in E\left(D_{i}\right)$, from some vertex $v_{j} \in S(x)$ to $v_{k} \in S(y)$. Because of the values of the labels of $x$ and $y$, it follows that $v_{j} \notin M$ and $v_{k} \in M$. Examine the following alternatives for the pair $v_{j}, v_{k}$. When $\left(v_{j}, v_{k}\right) \in E(G)$, the conditions $v_{j} \notin M$ and $v_{k} \in M$ imply $v_{j} \in N_{G}\left(v_{i}\right)$. By applying (1.1) and (1.2), we conclude that $\left(v_{j}, v_{k}\right) \notin E\left(D_{i}\right)$, a contradiction. When $\left(v_{j}, v_{k}\right) \notin E(G)$ it follows that $v_{j} \notin N_{G}\left(v_{i}\right)$. The same contradiction $\left(v_{j}, v_{k}\right) \notin E\left(D_{i}\right)$ arises by (2.1) and (2.2). The conclusion is that no such edge $\left(v_{j}, v_{k}\right) \in E\left(D_{i}\right)$ may exist, and consequentely the above choice of vertices $x, y$ of $P_{i}$ is not possible. That is, no vertex labelled 1 in $P_{i}$ has any descendant labelled 0 . Consequentely, $I$ is in fact an ideal of $P_{i}$.

Conversely, let $I$ be an ideal of $P_{i}$. We show the existence of a module $M$ of $G$ containing $v_{i}$, that can be made to correspond to $I$. Let $x \in V\left(P_{i}\right)$ and $S(x)$ the expansion of $x$. Let $M=\left\{v_{i}\right\} \bigcup_{x \in I} S(x)$. We will conclude that $M$ is a module of $G$. If $I=\emptyset$ or $I=V\left(P_{i}\right)$ then $M$ is a trivial module of $G$ and the argument is complete. Otherwise, there exists $v_{j} \in V(G) \backslash M$, with $j \neq i$. If for any such $v_{j}, M \subseteq N_{G}\left(v_{j}\right)$ or $M \subseteq \bar{N}_{G}\left(v_{j}\right)$, then $M$ is indeed a module of $G$, again completing the argument. Otherwise, there is $v_{j} \in V(G) \backslash M$ such that $M \nsubseteq N_{G}\left(v_{j}\right)$ and $M \nsubseteq \bar{N}_{G}\left(v_{j}\right)$. Then we can choose $v_{k}, v_{l} \in M$, such that $v_{k} \notin N_{G}\left(v_{j}\right)$ and $v_{l} \in N_{G}\left(v_{j}\right)$. First, we examine if $i$ can be equal to $k$ or $l$. Suppose $i=k$. Then $v_{j} \notin N_{G}\left(v_{i}\right)$. Since $\left(v_{j}, v_{l}\right) \in E(G)$, by (1.1) and (1.2) we conclude that $\left(v_{j}, v_{l}\right) \in E\left(D_{i}\right)$. Denote by $x^{\prime} \in V\left(P_{i}\right)$ and $x^{\prime \prime} \in V\left(P_{i}\right)$ the reductions in $P_{i}$ of $S\left(v_{j}\right)$ and $S\left(v_{l}\right)$, respectively. Because $v_{j} \notin M$ and $v_{l} \in M$, we conclude that $x^{\prime} \notin I$ and $x^{\prime \prime} \in I$. However $\left(v_{j}, v_{l}\right) \in E\left(D_{i}\right)$. Hence $I$ can not be an ideal of $P_{i}$, a contradiction. The second alternative, $i=l$ is similar and can not occur too. Consequently, $i \neq k, l$.

The proof proceeds by examining all possibilities of contaiments in $N_{G}\left(v_{i}\right)$ and $\bar{N}_{G}\left(v_{i}\right)$ of the vertices $v_{j}, v_{k}, v_{l}$.

Case $1 v_{j}, v_{k} \in N_{G}\left(v_{i}\right)$

By (2.1) it follows that $\left(v_{j}, v_{k}\right),\left(v_{k}, v_{j}\right) \in E\left(D_{i}\right)$. Then $v_{j}$ and $v_{k}$ belong to a same strongly connected component of $D_{i}$. In this case, $v_{j}, v_{k} \in S(x)$, for some vertex $x$ of $P_{i}$. By the construction of $M$, if $x \in I$ then $v_{j}, v_{k} \in M$. The latter contradicts $v_{j} \notin M$. The opposite case, $x \notin I$, implies $v_{j}, v_{k} \notin M$, contradicting $v_{k} \in M$. Hence Case 1 can not occur.

Case $2 v_{j} \in N_{G}\left(v_{i}\right)$ and $v_{k} \in \bar{N}_{G}\left(v_{i}\right)$.

By $(2.2)$, it follows that $\left(v_{j}, v_{k}\right) \in E\left(D_{i}\right)$. If $v_{j}$ and $v_{k}$ belong to the same strongly connected component of $D_{i}$, a similar argument as the Case 1 , leads to a contradiction. When $v_{j}$ and $v_{k}$ belong to distinct components, let $x$ and $x^{\prime}$ represent the reductions in $P_{i}$ of $S\left(v_{j}\right)$ and $S\left(v_{k}\right)$, respectively. That is, $v_{j} \in S(x)$ and $v_{k} \in S\left(x^{\prime}\right)$. Consequentely, $x \notin I$ and $x^{\prime} \in I$. However, $\left(v_{j}, v_{k}\right) \in E\left(D_{i}\right)$ implies that $x$ is an ancestor of $x^{\prime}$ in $P_{i}$. The latter contradicts $I$ to be an ideal of $P_{i}$.

Case $3 v_{j} \in \bar{N}_{G}\left(v_{i}\right)$ and $v_{l} \in N_{G}\left(v_{i}\right)$

By (1.2) we conclude that $\left(v_{j}, v_{l}\right) \in E\left(D_{i}\right)$. A contradiction arises by applying an argument similar as in Case 2, with $v_{l}$ replacing $v_{k}$. 
Case $4 v_{j}, v_{l} \in \bar{N}_{G}\left(v_{i}\right)$

Applying (1.1) we conclude that $\left(v_{j}, v_{l}\right),\left(v_{l}, v_{j}\right) \in$ $E\left(D_{i}\right)$. Hence $v_{j}$ and $v_{l}$ belong to a same strongly connected component of $D_{i}$. Similarly as in Case 1, we conclude that the present situation can not occur too.

The above four cases cover all eight possibilities for containment in $N\left(v_{i}\right)$ of $v_{j}, v_{k}, v_{l}$. Since none of them can occur, we conclude that there is no triple $v_{j}, v_{k}, v_{l} \in V(G)$, with $v_{j} \notin M$ and $v_{k}, v_{l} \in M$, satisfying $v_{k} \notin N_{G}\left(v_{j}\right)$ and $v_{l} \in N_{G}\left(v_{j}\right)$. Therefore, $M$ is indeed a module of $G$.

Each of the two above described correspondences is the inverse of the other. This completes the proof of Theorem 1.

For example, in the graph $G$ of Figure 1, the correspondences between modules $M_{i}$ containing the vertex $v_{1}$ and ideals $I_{i}$ of the modular poset $P_{1}$ of $G$ relative to $v_{1}$ of Figure 4 are as follows:

$M_{0}=\left\{v_{1}\right\}$ corresponds to $I_{0}=\{\emptyset\}$,

$M_{1}=\left\{v_{1}, v_{2}\right\}$ corresponds to $I_{1}=\left\{x_{1}\right\}$,

$M_{2}=\left\{v_{1}, v_{2}, v_{3}, v_{4}\right\}$ corresponds to $I_{2}=\left\{x_{1}, x_{2}\right\}$,

$M_{3}=\left\{v_{1}, v_{2}, v_{7}\right\}$ corresponds to $I_{3}=\left\{x_{1}, x_{3}\right\}$,

$M_{4}=\left\{v_{1}, v_{2}, v_{3}, v_{4}, v_{7}\right\}$ corresponds to $I_{4}=$ $\left\{x_{1}, x_{2}, x_{3}\right\}$,

$M_{5}=\left\{v_{1}, v_{2}, v_{3}, v_{4}, v_{7}, v_{5}, v_{6}\right\}$ corresponds to $I_{5}=$ $\left\{x_{1}, x_{2}, x_{3}, x_{4}\right\}$.

Theorem 1 has shown a correspondence between the modules of $G$ containing $v_{i}$ and ideals of the corresponding modular poset of $G$. In the sequel, the interest is to characterize these posets. We use more notation.

Let $D$ be an acyclic digraph. A layer decomposition of $D$ is a sequence $L_{1}, \ldots, L_{t}$ of subsets $L_{k} \subseteq V(D)$, such that $\bigcup_{1 \leq k<t} L_{k}=V(D)$ and $L_{k}$ is the set of sources of the digraph $D \backslash L_{k}^{-}$, where $L_{1}^{-}=\emptyset$ and $L_{k}^{-}=\bigcup_{1<l<k} L_{l}$, for $k>1$.

Each $\bar{L}_{k}$ is called a layer of $D$. Clearly the layer decomposition of $D$ is unique. Say that the digraph $D$ is layered, when its layer decomposition $L_{1}, \ldots, L_{t}$ is such that $\left(v_{j}, v_{l}\right) \in E(D)$, for any $v_{j} \in L_{k}$ and $v_{l} \in L_{k+1}, 1 \leq k<t$.

Theorem 3 The following affirmatives are equivalent:

(i) $P_{i}$ is the modular poset of some graph $G$, relative to $v_{i} \in V(G)$, (ii) $P_{i}$ is a layered poset,

(iii) $P_{i}$ is the transitive closure of an acyclic bipartite tournament.

Proof: (i) $\Rightarrow$ (ii): Let $G$ be a graph, $v_{i} \in V(G)$, $D_{i}$ the modular digraph of $G$, relative to $v_{i}, C_{i}$ the condensation of $D_{i}$, and $P_{i}$ the corresponding modular poset of $G$. We have to show that $P_{i}$ is layered. We can classify the vertices of $P_{i}$ (and $C_{i}$ ) into three types as follows. Let $x \in V\left(P_{i}\right)$ and $S(x) \subseteq V\left(D_{i}\right)$ the expansion of $x$. Then

$$
x \text { is of type } \begin{cases}0 & \text { when } S(x) \subseteq N_{G}\left(v_{i}\right) \\ 1 & \text { when } S(x) \subseteq \bar{N}_{G}\left(v_{i}\right) \\ 2 & \text { otherwise }\end{cases}
$$

Similarly, say that a vertex $v_{j} \in S(x)$ is a type $l$ vertex of $D_{i}$, when $x$ is a type $l$ vertex of $P_{i}, 0 \leq l \leq 2$. Denote by $T_{l}$ the set of type $l$ vertices of $P_{i}$. The following proposition is useful.

Proposition 4 Let $x$ be a vertex of $C_{i}$. Then

$$
x \text { is of type }\left\{\begin{array}{lll}
0 & \Rightarrow & N_{C_{i}}(x)=T_{1} \cup T_{2} \\
1 & \Rightarrow & N_{C_{i}}(x)=T_{0} \cup T_{2} \\
2 & \Rightarrow & N_{C_{i}}[x]=V\left(C_{i}\right)
\end{array}\right.
$$

Proof: To prove the above fact, we can assume that $P_{i}$ has at least two vertices, otherwise the proposition is trivial. Let $x, y$ be distinct vertices of $P_{i}$. Consider the following alternatives.

Case 1: $x$ and $y$ are type 0.

Then $S(x), S(y) \subseteq N_{G}\left(v_{i}\right)$. Let $v_{j} \in S(x)$ and $v_{k} \in S(y)$. Examining (1.1) - (1.2) and (2.1) - (2.2), since $v_{j}, v_{k} \in N_{G}\left(v_{i}\right)$ we conclude that (2.1) gives $\left(v_{j}, v_{k}\right),\left(v_{k}, v_{j}\right) \in E\left(D_{i}\right)$. This implies that $v_{j}, v_{k}$ belong to the same strongly connected component of $D_{i}$, contradicting $x, y$ to be distinct. Hence alternative (2.1) does not occur. Consequently, $v_{k} \notin N_{D_{i}}\left(v_{j}\right)$. Since $v_{j}$ and $v_{k}$ are arbitrary vertices of $S(x)$ and $S(y)$, respectively, we conclude that there are no edges in $C_{i}$ between a vertex of $S(x)$ and another of $S(y)$. Consequently, $y \notin N_{C_{i}}(x)$.

Case 2: $x$ is type 0 and $y$ is type 1 .

Then $S(x) \subseteq N_{G}\left(v_{i}\right)$ and $S(y) \subseteq \bar{N}_{G}\left(v_{i}\right)$. Again, let $v_{j} \in S(x)$ and $v_{k} \in S(y)$. If $\left(v_{j}, v_{k}\right) \in E(G)$, applying (1.2) implies that $\left(v_{k}, v_{j}\right) \in E\left(D_{i}\right)$. When $\left(v_{j}, v_{k}\right) \notin$ $E(G),(2.2)$ leads to $\left(v_{j}, v_{k}\right) \in E\left(D_{i}\right)$. The conclusion is that $v_{k} \in N_{D_{i}}\left(v_{j}\right)$, meaning that $y \in N_{C_{i}}(x)$. 
Case 3: $x$ is type 0 and $y$ is type 2 .

We know that $S(x) \subseteq N_{G}\left(v_{i}\right)$, while $S(y) \nsubseteq N_{G}\left(v_{i}\right)$ and $S(y) \nsubseteq \bar{N}_{G}\left(v_{i}\right)$. Then we can choose $v_{j} \in S(x)$ and $v_{k} \in S(y)$, such that $v_{j} \in N_{G}\left(v_{i}\right)$ and $v_{k} \in$ $\bar{N}_{G}\left(v_{i}\right)$. Similarly as in Case 2, (1.2) and (2.2) imply that either $\left(v_{k}, v_{j}\right) \in E\left(D_{i}\right)$ or $\left(v_{j}, v_{k}\right) \in E\left(D_{i}\right)$. Consequentely, $v_{k} \in N_{D_{i}}\left(v_{j}\right)$, that is $y \in N_{C_{i}}(x)$.

Case 4: $x$ and $y$ are type 1 .

Similarly, as in Case 1, we obtain $y \notin N_{C_{i}}(x)$.

Case 5: $x$ is type 1 and $y$ type 2 .

Analogous to Case 3 , and we conclude that $y \in$ $N_{C_{i}}(x)$.

Case 6: $x$ and $y$ are type 2 .

That is, $S(x), S(y) \nsubseteq N_{G}\left(v_{i}\right)$ and $S(x), S(y) \subseteq$ $\bar{N}_{G}\left(v_{i}\right)$. This means that the following choice of vertices is possible. Let $v_{j} \in S(x)$, and $v_{k} \in S(y)$ satisfying $v_{j} \in N_{G}\left(v_{i}\right)$ and $v_{k} \in \bar{N}_{G}\left(v_{i}\right)$. This situation is again similar to Case 2, implying that $y \in N_{C_{i}}(x)$.

As the last step of the proof, look at all the above cases. Let $x \in V\left(P_{i}\right)$ be a type 0 vertex. Applying Cases 1,2 and 3 , we conclude that $N_{C_{i}}(x)=T_{1} \cup$ $T_{2}$. If $x$ is of type 1 , then Cases 2,4 and 5 lead to $N_{C_{i}}(x)=T_{0} \cup T_{2}$. The last alternative is that $x$ is type 2, which implies $N_{C_{i}}[x]=V\left(C_{i}\right)$, by Cases 3,5 and 6 . Proposition 4 is proved.

In the sequel, let $L_{1}, \ldots, L_{t}$ be a layer decomposition $L$ of $C_{i}$. Examine the types of vertices in $C_{i}$ belonging to a same layer or to consecutives layers in $L$. Let $x, y$ be distinct vertices of $C_{i}$. Suppose that $x, y$ belong to a same layer of $L$. Then they can not be adjacent in $C_{i}$. By Proposition 4, it follows that if $x$ is of type 0 , so is $y$; when $x$ is type of 1 , so is $y$; and if $x$ is of type 2 implics that y can not exist. Consequentely, there can be no layer formed by vertices of distinct types. Moreover, any type 2 vertex is the sole vertex in its layer. Then we can say that layer $L_{q}$ is of type $l$, when $L_{q}$ contains a type $l$ vertex, $0 \leq l \leq 2$. We study the alternatives when $x, y$ belong to consecutive layers of $L$. Suppose that the theorem is false, that is, $P_{i}$ is not a layered poset. Therefore, $C_{i}$ is not layered too. Then there exist $x \in L_{q}$ and $y \in L_{q+1}$, such that $(x, y) \notin E\left(C_{i}\right)$. Because $L$ is a layered decomposition, we know that $(y, x) \notin E\left(C_{i}\right)$. Then $y \notin N_{C_{i}}(x)$. Consequentely, neither layer $L_{q}$ nor $L_{q+1}$ can be of type 2. Since $L$ is a layer decomposition, there are $x^{\prime} \in L_{q}$ and $y^{\prime} \in L_{q+1}$, such that $\left(x^{\prime}, y^{\prime}\right) \in E\left(C_{i}\right)$. By Proposition 4 , the latter implies that $L_{q}$ and $L_{q+1}$ can not be both of a same type. Consequentely, $L_{q}$ is of type 0 and $L_{q+1}$ is of type 1 , or vice-versa. Again, by Proposition 4 , this implies that $(x, y) \in E\left(C_{i}\right)$, contradicting our inicial assumption. Therefore, $C_{i}$ is layered and consequentely so is $P_{i}$.

(ii) $\Rightarrow$ (iii): Let $P_{i}$ be a layered poset. The proof is to construct a bipartite tournament $B_{i}$, such that $P_{i}$ is the transitive closure of $B_{i}$. Let $L_{1}, \ldots, L_{t}$ be the layer decomposition $L$ of $P_{i}$. Denote by $B_{i}$ the subdigraph obtained from $P_{i}$ by removing the edges of $P_{i}$ between vertices belonging to layers $L_{q}, L_{p}$, such that $q$ and $p$ are both odd or both even. The vertices of $B_{i}$ can be partitioned into two subsets $L^{\prime}, L^{\prime \prime}$, the first formed by the odd layers and the second by the even layers of $L$.

By construction, $B_{i}$ has no edges between vertices of the same subset $L^{\prime}$ or $L^{\prime \prime}$. On the other hand, since $P_{i}$ is a layered poset, $x \in L^{\prime}$ and $y \in L^{\prime \prime}$ imply that $x$ and $y$ are adjacent in $P_{i}$, and therefore adjacent in $B_{i}$. That is, $B_{i}$ is a bipartite tournament. Since $P_{i}$ is acyclic, so is $B_{i}$. In addition, all the edges of $E\left(P_{i}\right) \backslash E\left(B_{i}\right)$ are necessarily implied by transitivity. Consequentely, $P_{i}$ is the transitive closure of $B_{i}$. That is, (ii) $\Rightarrow$ (iii).

(iii) $\Rightarrow$ (i): By hipothesis, $P_{i}$ is the transitive closure of an acyclic bipartite tournament $B_{i}$. We have to prove that $P_{i}$ is the modular poset of some graph $G$, relative to a vertex $v_{i} \in V(G)$. With this purpose, we construct an undirect graph $G$, having $B_{i}$ as its modular digraph, relative to $v_{i}$. Define $V(G)=V\left(B_{i}\right) \cup\left\{v_{i}\right\}$, where $v_{i} \notin V\left(B_{i}\right)$. The edges of $G$ are defined in the sequel. Let $V_{0} \cup V_{1}=V\left(B_{i}\right)$ be a bipartition of the vertices of $B_{i}$. The vertices of $G$ adjacent to $v_{i}$ are defined as being all of $V_{0}$ and none of $V_{1}$. The set $V_{0}$ is a clique of $G$, while $V_{1}$ is an independent set of $G$. It remains to define the edges $\left(v_{j}, v_{k}\right)$ of $G$, such that $v_{j} \in V_{0}$ and $v_{k} \in V_{1}$. For each pair $v_{j}, v_{k}$, where $v_{j} \in V_{0}$ and $v_{k} \in V_{1}$, we know that $B_{i}$ contains exactly one of the directed edges $\left(v_{j}, v_{k}\right)$ or $\left(v_{k}, v_{j}\right)$. The decision of whether or not $\left(v_{j}, v_{k}\right)$ will be included as an undirected edge of $G$ depends on its direction in $B_{i}$, as follows. Include $\left(v_{j}, v_{k}\right)$ in $E(G)$ if and only if its direction in $B_{i}$ is from $v_{k}$ to $v_{j}$. The construction of $G$ is complete.

The next task is to show that $B_{i}$ is precisely the modular digraph $D_{i}$ of $G$ relative to $v_{i}$. Clearly, $V\left(D_{i}\right)=V\left(B_{i}\right)$, as both of them are equal to $V(G) \backslash$ $\left\{v_{i}\right\}$. By the construction of $G, N_{G}\left(v_{i}\right)=V_{0}$ and 
$\bar{N}_{G}\left(v_{i}\right)=V_{1}$. Since $V_{0}$ is a clique in $G,(1.1)$ and (1.2) assure that $D_{i}$ has no edges between two vertices of $V_{0}$. Similarly, from (2.1) and (2.2) we conclude that there are no edges in $D_{i}$ between two vertices of $V_{1}$. Finally, for each pair $v_{j} \in V_{0}$ and $v_{k} \in V_{1},(1.2)$ assures that when $\left(v_{j}, v_{k}\right) \in E(G), D_{i}$ contains the directed edge $\left(v_{k}, v_{j}\right)$. Similarly, from $(2.2)$ we conclude that whenever $\left(v_{j}, v_{k}\right) \notin E(G)$, it follows $\left(v_{j}, v_{k}\right) \in E\left(D_{i}\right)$. This completes the description of $D_{i}$. Observe that $D_{i}$ is a bipartite digraph, with bipartition $V_{0} \cup V_{1}$. In addition, for $v_{j} \in V_{0}$ and $v_{k} \in V_{1}, D_{i}$ contains either the edge $\left(v_{j}, v_{k}\right)$ or $\left(v_{k}, v_{j}\right)$, its direction being the same as in $B_{i}$. Consequentely, $D_{i}=B_{i}$. Since $B_{i}$ is acyclic, so is $D_{i}$. Therefore the condensation $C_{i}$ of $D_{i}$ coincides with $D_{i}$. Because $P_{i}$ is the transitive closure of $B_{i}$, we conclude that $P_{i}$ is the transitive closure of $C_{i}$. Hence $P_{i}$ is the modular poset of $G$, relative to $v_{i}$. This completes the proof of Theorem 3.

\section{Applications}

In the last section, it has been shown that the set of modules of a graph $G$, containing a vertex $v_{i} \in V(G)$, can be described by the modular poset of $G$, relative to $v_{i}$. In this section, we present applications of this representation.

\subsection{Enumerating the modules}

The first application is on the enumeration of the modules of a graph $G$. We present methods for generating and counting the modules of $G$. The following simple proposition is useful.

Proposition 5 Let $P$ be a layered poset with layers $L_{1}, \ldots, L_{t}$. Then there exists an one-to-one correspondence between non-empty ideals $I$ of $P$ and nonempty subsets $L_{k}^{\prime} \subseteq L_{k}$, for all $1 \leq k \leq t$. Moreover $I=L_{k}^{\prime} \bigcup_{1 \leq \ell<k} L_{\ell}$.

Proof: Let $I$ be an ideal of $P$, and $k$ the largest index of a layer $L_{k}$ of $P$, such that $I \cap L_{k} \neq \emptyset$. Then $L_{1}, \ldots, L_{k-1} \subseteq I$. Let $L_{k}^{\prime}=I \backslash \bigcup_{1<\ell<k} L_{l}$. Choose $L_{k}^{\prime} \subseteq L_{k}$ to correspond to $I$. Conversely, by hypothesis $L_{k}^{\prime} \neq \emptyset$ and $L_{k}^{\prime} \subseteq L_{k}$, for some $1 \leq k \leq t$. Then $I=L_{k}^{\prime} \bigcup_{1<\ell<k} L_{\ell}$ is clearly an ideal, precisely the one corresponding to $L_{k}^{\prime}$. 口

Using Theorem 1, a possible method for enumerating the modules of $G$ is to choose an ordering $v_{1}, \ldots, v_{n}$ of the vertices of $G$, and iteratively enumerate the modules of $G$ containing $v_{i}$, except those also containing any of the preceding vertices $v_{1}, \ldots, v_{i-1}$. With the purpose of applying this idea let $P_{i}$ be the modular poset of $G$ relative to $v_{i}$. Define the modular poset $P_{i}^{\prime}$ relative to $v_{1}, \ldots, v_{i}$, as follows. If $i=1$ then $P_{i}^{\prime}=P_{i}$, and for $i>1 P_{i}^{\prime}=P_{i} \backslash \bigcup_{1<j<i} N_{P_{i}}^{+}[x]$, where $x \in V\left(P_{i}\right)$ is the reduction of $S\left(v_{j}\right)$ in $P_{i}$. Clearly, $P_{i}^{\prime}$ is also a layered poset. The next proposition describes a correspondence between all modules of $G$ and ideals of $P_{i}^{\prime}$.

Proposition 6 Let $G$ be a graph, $v_{1}, \ldots, v_{i}$ a sequence of vertices of it, and $P_{i}^{\prime}$ the modular poset of $G$ relative to $v_{1}, \ldots, v_{i}$. Then there exists a one-to-one correspondence between the ideals of $P_{i}^{\prime}$ and the modules of $G$, containing $v_{i}$ and not any of the vertices $v_{1}, \ldots, v_{i-1}$.

Proof: Let $M$ be a module of $G$ containing $v_{i}$ and not containing any $v_{1}, \ldots, v_{i-1}$. By Theorem $1, P_{i}$ has an ideal $I$ corresponding to $M$. Moreover, $M=$ $\left\{v_{i}\right\} \bigcup_{x \in I} S(x)$. We show that $I$ is also an ideal of $P_{i}^{\prime}$ and can be chosen as corresponding to $M$. If $i=1$, this is trivial. Let $i>1$ and consider the alternatives.

Case 1: $I \nsubseteq \subset\left(P_{i}^{\prime}\right)$

Then any vertex $x \in I \backslash V\left(P_{i}^{\prime}\right)$ is such that $x \in N_{P_{i}}^{+}[y]$, where $y$ is the reduction in $P_{i}$ of $S\left(v_{j}\right) \subseteq V(G)$, for some $1 \leq j<i$. Because $v_{j} \notin M$, it follows that $y \notin I$. This means that $I$ is not an ideal of $P_{i}$, since $x \in I$ has an ancestor $y \notin I$ in $P_{i}$. This contradiction leads to the conclusion that this case does not occur.

Case 2: $I \subseteq V\left(P_{i}^{\prime}\right)$

Then $I$ is also an ideal of $P_{i}^{\prime}$, since the sets of ancestors of the vertices of $I$ are the same in $P_{i}$ and $P_{i}^{\prime}$.

Conversely, let $I$ be an ideal of $P_{i}^{\prime}$. Then $I$ is also an ideal of $P_{i}$. By theorem $1, M=\left\{v_{i}\right\} \bigcup_{x \in I} S(x)$ is a module of $G$ containing $v_{i}$. Since $I \subseteq V\left(P_{i}^{\prime}\right), I$ does not contain any vertex which is the reduction in $P_{i}$ of $S\left(v_{j}\right) \subseteq V(G)$, for some $1 \leq j<i$. Consequently. $v_{j} \notin M$. That is, $M$ is a module of $G$ containing $v_{i}$ and not $v_{1}, \ldots, v_{i-1}$. Therefore, $M$ is the module of $G$ corresponding to the ideal $I$ of $P_{i}^{\prime}$.

The algorithm for generating all modules of a graph $G$ follows directly from Proposition 6 . Choose an arbitrary ordering $v_{1}, \ldots, v_{n}$ of the vertices of $G$. For $1 \leq i \leq n$ construct the modular poset $P_{i}^{\prime}$, relative to $v_{1}, \ldots, v_{i}$. Generate all ideals $I$ of $P_{i}^{\prime}$ and compute the corresponding modules $\left\{v_{i}\right\} \bigcup_{x \in I} S(x)$ of $G$. Generating all the ideals of $P_{i}^{\prime}$ is equivalent to generating all the subsets of the layers of $P_{i}^{\prime}$, by Theorem 3 and Proposition 5. The latter step requires constant amortized time. The overall time bound of the algorithm 
is $O\left(n^{3}+\mu\right)$, where $\mu$ is the total number of modules of $G$. The (worst-case) delay complexity is $O\left(n^{2}\right)$.

As for the counting problem, the number of distinct modules of $G$ can be computed using the expression given by the proposition below. The proof of it follows directly from Theorems 1, 3 and Propositions 5 and 6 .

Proposition 7 Let $G$ be a graph, $\mu$ the number of modules of $G$, and $v_{1}, \ldots, v_{n}$ an ordering of its vertices. Denote by $L_{i_{1}}, \ldots, L_{i_{t_{i}}}$ the layer decomposition of the modular poset of $G$, relative to $v_{1}, \ldots, v_{i}$. Then

$$
\mu=n+\sum_{1 \leq i \leq n} \sum_{1 \leq k \leq t_{i}}\left(2^{\left|L_{i_{k}}\right|}-1\right)
$$

The corresponding counting algorithm requires $O\left(n^{3}\right)$ steps.

\subsection{Finding special modules}

Consider the problem of finding a module of a graph $G$ satisfying a given property. Below, we describe solutions for two distinct cases. The first is to find a maximal module of $G$ satisfying an hereditary property, while the second corresponds to finding a non-trivial connected module of a graph, if existing.

We use more notation. Let $G$ be graph, $v_{i} \in$ $V(G), P_{i}$ the modular poset of $G$, relative to $v_{i}$, and $L_{1}, \ldots, L_{t}$ the layer decomposition of $P_{i}$. Let $S(X)=\bigcup_{x \in X} S(x)$ for $X \subseteq V\left(P_{i}\right)$. Denote $S_{0}=\left\{v_{i}\right\}$ and $S_{k}=S_{k-1} \bigcup S\left(L_{k}\right)$. Finally, $S_{k}^{i} \subseteq S_{k}$ represents the subset of vertices forming the connected component containing $v_{i}$ of the subgraph induced in $G$ by $S_{k} \subseteq V(G)$.

\subsubsection{Finding modules satisfying hereditary properties}

A property $\pi$ on graphs is a collection of graphs, closed under isomorphism. When a graph $G$ belongs to $\pi$, say that $V(G)$ satisfies $\pi$. When $\pi$ is closed under induced subgraphs, say that it is an hereditary property.

The following proposition describes the maximal modules of a graph $G$ containing a vertex $v_{i} \in V(G)$ and satisfying an hereditary property $\pi$. Examples of such properties are planar graphs, chordal graphs, bipartite graphs, and so on.

Proposition 8 Let $G$ be a graph, $v_{i} \in V(G)$, and $L_{1}, \ldots, L_{t}$ the layer decomposition of the modular poset of $G$, relative to $v_{i}$. Let $\pi$ be an hereditary property satisfied by $\left\{v_{i}\right\}$. For $M \subseteq V(G), M$ is a maximal module of $G$ containing $v_{i}$ and satisfying $\pi$ if and only if $M$ satisfies $\pi$ and

$$
M=V(G), \text { or }
$$

$M=S \cup S\left(L_{k}^{\prime}\right)$, where $L_{k}^{\prime} \subseteq L_{k}, L_{k}^{\prime} \neq L_{k}$, for some $1 \leq k \leq t$, and

$M \cup\{v\}$ does not satisfy $\pi$, for all $v \in S\left(L_{k} \backslash L_{k}^{\prime}\right)$.

The above proposition leads to the following algorithm for finding a maximal module of $G$, containing $v_{i} \in V(G)$ and satisfying a given hereditary property $\pi$, where $\left\{v_{i}\right\} \in \pi$.

In the initial step, given $G$ and $v_{i}$, construct $D_{i}$ and $P_{i}$, find the layer decomposition $L_{1}, \ldots, L_{t}$ of $P_{i}$, define $k=1, \ell=0$ and $M=\left\{v_{i}\right\}$. In the general step, for each $v \in L_{k}$, if $M \cup\{v\}$ satisfies $\pi$ then include $v$ in $M$, otherwise set $\ell$ to 1 . After all vertices of $L_{k}$ have been examined, if $k=t$ or $\ell=1$, stop: $M$ is the desired module. Otherwise, increase $k$ by 1 and repeat the general step.

The complexity of the algoritm is $O\left(n^{2}+n C_{\pi}\right)$, where $C_{\pi}$ is the complexity of verifying whether $G$ satisfies $\pi$.

\subsubsection{Finding a connected non-trivial module}

The property connected graphs is not hereditary. Therefore finding a connected non-trivial module of a graph can not be solved by Proposition 8 . We describe below a method for finding such a module. We use an additional concept.

Let $G$ be a connected graph, $A, B \subseteq V(G)$ and $A \cap$ $B=\emptyset$. Say that $B$ separates $A$ in $G$ when the vertices of $A$ belong to more than one connected component of $G \backslash B$.

The following proposition describes the non-trivial connected modules of $G$.

Proposition 9 Let $G$ be a graph, $|V(G)| \geq 3, v_{i} \in$ $V(G)$ and $L_{1}, \ldots, L_{t}$ the layer decomposition of the modular poset of $G$, relative to $v_{i}$. There exists a connected non-trivial module of $G$, containing $v_{i}$, if and only if

(a) $\left\{v_{i}\right\} \neq S_{1}^{i} \neq V(G)$, or

(b) $S_{k-1} \subseteq S_{k}^{i} \neq V(G)$, for some $k>1$, or

(c) $S_{t}^{i}=V(G), t>1$, and $\{v\}$ does not separate $S_{t-1}$ in $G$, for some $v \in S\left(L_{t}\right)$.

Proof: Let $M$ be a non-trivial connected module of $G$, containing $v_{i}$. By Proposition $5, M$ is of the form $\left\{v_{i}\right\} \bigcup_{x \in L_{k}^{\prime}} S(x)$, for some $k$ and subset $L_{k}^{\prime} \subseteq L_{k}$. That is, $S_{k-1} \subseteq M$. Since $M$ is connected it follows that $M \subseteq S_{k}^{i}$. Consequently, $k=1$ implies 
$\left\{v_{i}\right\} \neq S_{1}^{i} \neq V(G)$, otherwise $M$ is trivial. That is, (a) is valid. Suppose $1<k<t$. Then $S_{k}^{i} \neq V(G)$ and (b) holds. Finally, let $k=t>1$. Examine the following two alternatives. If $S_{t}^{i} \neq V(G)$ then case (b) occurs again. Consider $S_{t}^{i}=V(G)$. Since $M$ is not trivial, there exists $B \subseteq S\left(L_{t}\right)$, such that $B \cap M=\emptyset$ and $B \neq \emptyset$. Because $S_{t-1} \subseteq M$ it follows that $B$ can not separate $S_{t-1}$ in $G$. Consequently, any subset $B^{\prime} \subseteq B$ also does not separate $S_{t-1}$ in $G$. That is, any $v \in B$ is such that $\{v\}$ does not separate $S_{t-1}$, meaning that condition (c) occurs. Hence $M$ being a non-trivial connected module of $G$ implies that at least one of the conditions (a), (b) or (c) occurs. The proof of the converse consists of exhibiting a module $M$ having the desired properties, whenever one of the conditions (a), (b) or (c) holds.

If (a) is verified then $S_{1}^{i}$ is a connected non-trivial module of $G$. So is $S_{k}^{i}$, when (b) occurs. Finally, suppose that (c) is true. In this case, the vertices of the connected component containing $v_{i}$, of the subgraph induced by $V(G) \backslash\{v\}$ form a non-trivial connected module of $G$.

The above proof leads directly to an algorithm for finding a connected non-trivial module of $G$, or reporting that one does not exist. In the worst situation, it might be necessary to check conditions (b) and (c) $O(n)$ times. Therefore the complexity of the algorithm is $O(n m)$.

\section{Conclusions}

We have presented a characterization of the modules of an undirected graph $G$, in terms of ideals of certain posets. As applications of it, we have described algorithms for (i) generating all the $\mu$ modules of $G$, (ii) counting the $\mu$ modules of $G$, (iii) finding a maximal module of $G$ containing $v_{i} \in V(G)$ and satisfying an hereditary property $\pi$, and (iv) finding a non-trivial connected module of $G$. The complexities of the algorithms are (i) $O\left(n^{3}+\mu\right)$, (ii) $O\left(n^{3}\right)$, (iii) $O\left(n^{2}+n C_{\pi}\right)$, where $C_{\pi}$ is the complexity of verifying whether $G$ satisfies $\pi$, and (iv) $O(n m)$.

Acknowledgements We are grateful to Luerbio Faria for helping us with the figures:This research was partially supported by CNPq and Pronex

\section{References}

[1] B. Buer and R. H. Mohring. A fast algorithm for the decomposition of graphs and posets. Math. Oper. Res. 8 170-184, 1983.
[2] A. Cournier and M. Habib. A new linear algorithm for modular decomposition. in Sophie Tison, ed., CAAP'94: 19th International Colloquium, Lectures Notes in Computer Science Edinburgh, UK, 68-82, 1994.

[3] E. Dahlhaus, J. Gustedt and R. M. McConnel. Efficient and pratical modular decomposition. Proceedings of the Eight Annual ACM-SIAM Symposium on Discrete Algorithms 26-35, 1997.

[4] A. Ehrenfeucht, H. N. Gabow, R. M. McConnel and S. J. Sullivan. An $O\left(n^{2}\right)$ divide-and-conquer algorithms for the prime tree decomposition of two-structures and modules decompositions of graphs. J. of Algorithms 16 283-294, 1994.

[5] T. Gallai. Transitiv orientierbare graphen. Acta math. Acad. Sci. hungar. 18, 25-66, 1967.

[6] M. C. Golumbic. Algorithmic Graph Theory and Perfect Graphs. Academic press, New York, 1980.

[7] M. Habib and M. C. Maurer. On the X-join decomposition for undirected graphs. Discrete Appl. Math. 1 201-207, 1979.

[8] D. Kelly. Comparability graphs. in I. Rival, ed. Graphs and Orders, Reidl, Boston, 3-40, 1985.

[9] R. M. McConnel and J. Spinrad. Linear-time modular decomposition and efficient transitive orientation of comparability graphs. in Proceedings of the Fifth Annual ACM-SIAM Symposium on Discrete Algorithms 536-545, 1994.

[10] R. M. McConnel and J. Spinrad. Linear -time transitive orientation. in Proceedings of the Eight Annual ACM-SIAM Symposium on Discrete Algorithms 19-25, 1997.

[11] R. M. McConnel and J. Spinrad. Modular decomposition and transitive orientation. Available at http://www.cs.amherst.edu/ross/work.html, 1997.

[12] J. H. Miller and J. Spinrad. Incremental modular decomposition. J. Assoc. Comput. mach. 36 119, 1989.

[13] R. H. Mohring. Algoritmic aspects of comparability graphs and interval graphs. in I. Rival, ed.Graphs and Orders, Reidl, Boston, 41-101, 1985.

[14] J. Spinrad. $P_{4}$ trees and substitution decomposition. Discrete Appl. Math. 39 263-291, 1992. 